\title{
Numerical Solution of the Coupled Viscous Burgers' Equation Using Differential Quadrature Method Based on Fourier Expansion Basis
}

\author{
Masho Jima, Alemayehu Shiferaw, Ali Tsegaye \\ Department of Mathematics, Jimma University, Jimma, Ethiopia \\ Email: maashookoo.reemii@gmail.com, abelhaim@gmail.com
}

How to cite this paper: Jima, M., Shiferaw, A. and Tsegaye, A. (2018) Numerical Solution of the Coupled Viscous Burgers' Equation Using Differential Quadrature Method Based on Fourier Expansion Basis. Applied Mathematics, 9, 821-835.

https://doi.org/10.4236/am.2018.97057

Received: December 18, 2017

Accepted: July 27, 2018

Published: July 30, 2018

Copyright $\odot 2018$ by authors and Scientific Research Publishing Inc. This work is licensed under the Creative Commons Attribution International License (CC BY 4.0).

http://creativecommons.org/licenses/by/4.0/

\begin{abstract}
The differential quadrature method based on Fourier expansion basis is applied in this work to solve coupled viscous Burgers' equation with appropriate initial and boundary conditions. In the first step for the given problem we have discretized the interval and replaced the differential equation by the Differential quadrature method based on Fourier expansion basis to obtain a system of ordinary differential equation (ODE) then we implement the numerical scheme by computer programing and perform numerical solution. Finally the validation of the present scheme is demonstrated by numerical example and compared with some existing numerical methods in literature. The method is analyzed for stability and convergence. It is found that the proposed numerical scheme produces a good result as compared to other researcher's result and even generates a value at the nodes or mesh points that the results have not seen yet.
\end{abstract}

\section{Keywords}

Differential Quadrature Method, Fourier Expansion, Coupled Viscous, Coupled Viscous Burgers' Equation, Initial and Boundary Conditions

\section{Introduction}

\subsection{Background of the Study}

In the field of computational mathematics, numerical methods are most widely utilized to solve equations arising in the field of physics, engineering and other sciences. Numerical analysis plays a significant role and helps us to find an ap- 
proximate solution for problems which are difficult to solve analytically. The design and computation of the numerical algorithm is one of the mathematical challenges faced nowadays, but scientists in the field of computational mathematics are trying to develop numerical methods by using of computers for further application. One of those numerical methods is quadrature method.

A differential quadrature method is a numerical method for evaluating derivatives of sufficiently smooth function as proposed by [1]. The basic idea of differential quadrature came from Gauss Quadrature [2], which is a useful numerical integration method. Gauss Quadrature is characterized by approximating a definite integral with weighting sum of integrand value of a group at Gauss point. Extending it to find the derivatives of various orders of sufficiently smooth function gives rise to DQ [1]. In other words the derivatives of smooth function are approximated with weighted sum of the function values at a group of so called nodes. Differential quadrature can be formulated through either approximation theory or solving a system of linear equations. However, the rapid development over the recent years on problems involving nonlinear, discontinuity, multiple scales, singularity and irregularity are challenges in the field of computational science and engineering of the various numerical solutions. DQ method has distinguished themselves because of their high accuracy, straight forward implementation and generality in variety of problems [3].

\section{Coupled Viscous Burgers' Equation}

Coupled Viscous Burgers' equation is the nonlinear partial differential equation, wildly applicable in the theory of shock waves, mathematical modeling of turbulent fluid and in continuous stochastic processes.

And this equation has a wide application in the various areas of applied mathematics, such as fluid mechanics, nonlinear acoustic gas dynamics and traffic flow. The coupled viscous Burgers' equation was studied for the first time by [4] to model polydisperse sedimentation or evaluate scaled volume concentration of two kinds of particles in fluid suspensions or colloid under the effect of gravity.

The viscous Burgers' equation was presented in 1940 and in 1950 Hopf and in 1951 Cole independently introduced the method that has come to be known as the Cole-Hopf transformation to solve the Viscous Burgers' Equation [3].

The Coupled Viscous Burgers' Equation which is a nonlinear partial differential equation of the form

$$
\begin{gathered}
u_{t}-u_{x x}+\eta u u_{x}+\alpha(u v)_{x}=0, x \in[a, b], t \in[0, T] \\
v_{t}-v_{x x}+\varepsilon v v_{x}+\beta(u v)_{x}=0, x \in[a, b], t \in[0, T]
\end{gathered}
$$

with initial condition

$$
u(x, 0)=\phi_{1}(x), v(x, 0)=\phi_{2}(x), 0 \leq x \leq 1
$$

The boundary condition

$$
\begin{gathered}
u(a, t)=f_{1}(a, t), \quad v(a, t)=g_{1}(a, t) \\
u(b, t)=f_{2}(b, t), \quad v(b, t)=g_{2}(b, t), 0 \leq t \leq T
\end{gathered}
$$


where $\eta, \varepsilon, \alpha$ and $\beta$ are constants depending on the system parameters.

\section{Differential Quadrature Method (DQM)}

Differential quadrature method first introduced by [1] is one of the most efficient numerical method to solve partial differential equations. The key procedure in differential quadrature approximation is to determine the weighting coefficient.

[5] and [6] obtain explicit formulations to compute the weighting coefficient of the first and second order derivative. [3] presented a simple algebraic formulation to compute the weighting coefficient of the first order derivative without any restriction on the choice of the grid points and a recurrence relationship to compute the weighting coefficient of the DQ method and its applications were rapidly developed after the late 1980s, thanks to the innovative work in the computation of the weighting coefficients by other researchers and the author. As a result, the DQ method has emerged as a powerful numerical discretization tool in the past decade. As compared to the conventional low order finite difference and finite element methods, the DQ method can obtain very accurate numerical results using a considerably smaller number of grid points and hence requiring relatively little computational effort. So far, the DQ method has been efficiently employed in a variety of problems in engineering and physical sciences.

Based on the analysis of function approximation and the analysis of linear vector space, the $D Q$ method can be classified as polynomial-based DQ (PDQ) and Fourier series expansion-based DQ (FDQ) methods. PDQ is usually applied to non-periodic problems while FDQ can be applied to both periodic and non-periodic problems. But its performance for periodic problems is much better. The details of PDQ and FDQ methods and their application in engineering can be referred to the book of [3] which is the first book in the area to systematically describe the DQ method and its application in engineering.

\section{Fourier Expansion Basis}

The polynomial approximation is suitable for most of the engineering problems, but for some problems, especially for those with periodic behaviors, Fourier series expansion could be a better choice for the true solution instead of polynomial approximate the interval $[0,2 \pi][7]$ and [4], the Fourier series expansion can be given by

$$
f(x)=a_{0}+\sum_{k=1}^{\infty}\left(c_{k} \cos k x+d_{k} \sin k x\right)
$$

where the coefficient $a_{0}, c_{k}$ and $d_{k}$ are expressed as

$$
\begin{gathered}
a_{0}=\frac{1}{2 \pi} \int_{0}^{2 \pi} f(x) \mathrm{d} x, \\
c_{k}=\frac{1}{\pi} \int_{0}^{2 \pi} f(x) \cos k x \mathrm{~d} x \\
d_{k}=\frac{1}{\pi} \int_{0}^{2 \pi} f(x) \sin k x \mathrm{~d} x,
\end{gathered}
$$


For practical application, the truncated Fourier series expansion is usually used. Thus Equation (1.4) can be described as

$$
f(x)=F_{N+1}=a_{0}+\sum_{k=1}^{N}\left(c_{k} \cos k x\right)+\sum_{k=1}^{N}\left(d_{k} \sin k x\right)
$$

Moreover, the convergence of the above Equation (1.8) of $f(x)$ as $N \rightarrow \infty$ is guaranteed by Weirstrass's second theorem. i.e.

The function $f(x)$ be continuous on the interval $[0,2 \pi]$. Then for any $\varepsilon>0$ there exists an integer $n$ and a trigonometric $S_{n}$ such that the inequality

$$
\max _{x \in[0,2 \pi]}\left|f(x)-s_{N}(x)\right|<\varepsilon \text { is satisfied for all values of } x \text {. }
$$

where

$$
s_{n}(x)=a_{o}+\sum_{k=1}^{N} a_{k} \cos k x+\sum_{k=1}^{N} b_{k} \sin k x
$$

\subsection{Statement of the Problem}

Consider the Coupled Viscous burgers' Equation in Equations (1.1)-(1.3) is a nonlinear partial differential equation, by using Fourier basis based on weighted average differential quadrature method one can expect to find its' solution numerically by using some approximation method.

In this regard [4] presented numerical simulations for the Coupled Viscous Burgers' Equation and compared the results with experimental data recently; [9] proposed a Fourier Pseudospectral method for solving Coupled Viscous Burgers' Equation; [10] and [11] applied differential quadrature method to solve the viscous Burgers' Equation; [7] use finite difference and cubic spline finite element methods to solve Burgers' equation; [10] and [11] used polynomial Differential quadrature method for numerical solution of coupled viscous Burger' equations; [12] transform the Burgers' equation to linear heat equation using Hopf-Cole transformation and then use explicit finite difference and exact explicit finite difference method to solve the transformed linear heat equation with Neumann boundary condition; [13] solve one dimensional Burgers' Equation by using differential quadrature method based on Fourier Expansion basis; [10] used cubic B-spline collocation scheme based on Crank-Nicolson formulation for time integration and cubic B-spline functions for space integration by linearizing the nonlinear terms to solve coupled viscous Burgers' Equation.

In this study, we look for the solution of coupled viscous Burger' equation by applying the differential quadrature method based on Fourier Expansion basis. As a result, this study attempted to answer the following basic research questions.

1) How do we describe the differential quadrature method based on Fourier Expansion basis for coupled viscous Burgers' equation?

2) How the present method is applied to solve coupled viscous Burgers' equation?

3) To what extent the proposed method is approximate the exact solution? 


\subsection{Objectives of the Study}

\subsubsection{General Objective}

The general objective of this study is to find the numerical solution of coupled viscous burger equation using differential quadrature method based on Fourier expansion basis.

\subsubsection{Specific Objectives}

The specific objectives of the present study are:

1) To describe the differential quadrature method based on fourier expansion basis in solving coupled viscous Burgers' equation numerically.

2) To solve the coupled viscous Burgers' equation using differential quadrature method based on a Fourier expansion.

3) To determine the accuracy that the proposed method approximate the exist solutions.

\subsection{Significance of the Study}

The outcomes of this study have the following importance to:

1) Find an alternative numerical solution of coupled viscous Burgers' Equation.

2) Apply differential quadrature method based on Fourier expansion basis to find the solution for some practical problems like coupled viscous Burgers' equation.

\subsection{Delimitation of the Study}

This study was delimited to the numerical solution of the coupled viscous Burgers' equation.

The study also delimited to the practically most important (linear) differential quadrature based on Fourier expansion that are of the same type as differential quadrature and Fourier expansion formulas, i.e., linear combinations of weighted differential function evaluations and their error analysis for quadrature.

\section{Methodology}

\subsection{Study Area and Period}

The study was conducted in Jimma University under the department of mathematics from September 2016 G.C. to September 2017 G.C. Conceptually the study focus on the solution of coupled viscous Burgers' equation using differential quadrature method based on Fourier expansion basis.

\subsection{Study Design}

This study was employed mixed-design (documentary review design and experimental design) on the coupled viscous Burgers' equation type.

\subsection{Source of Information}

The relevant sources of information for this study is books, published articles 
and related studies from internet and the experimental results were obtained by writing MATLAB code for the present methods.

\subsection{Mathematical Procedures}

The study is an experimental as it evolves entirely laboratory work with the help of computer and MATLAB software. Farther, important materials for the study were collected by the researcher using the documentary analysis. The required numerical data was collected by coding and running using MATLAB software to get the numerical results and table of some examples that have exact solution, to show the validity and efficiency of the method. Hence, in order to achieve the stated objectives, the study was followed the procedures

1) Problem preparation or formulation.

2) Discretizing the space variable.

3) Replacing the partial differential equation (or the Coupled Viscous Burgers' Equation) by using differential quadrature method based on Fourier expansion basis to obtain a system of first order ordinary differential equations.

4) The obtained systems of ordinary differential equations was solved by classical fourth order Runge-Kutta method.

5) Validating the schemes using numerical examples.

6) Writing MATLAB code for the method to solve the systems obtained.

\section{Result and Discussion}

\subsection{Preliminaries}

\subsubsection{Differential Quadrature Method}

In seeking an efficient discretization technique to obtain accurate numerical solution using a considerably small number of grid points, [1] and [14] introduced the method of differential quadrature (DQ).where a partial derivative of a function with respect to a coordinate direction is expressed as a linear weighted sum of all the functional values at all mesh points along that direction. The key to DQ is to determine the weighting coefficient for the discretization of a derivative of any order. [1] suggested two methods to determine the weighting coefficients of first order derivative. The first method is based on an ill-conditioned algebraic equation system and the second method uses a simple algebraic formulation, but the coordinates of the grid points are fixed by the roots of the shifted Legendre polynomial. In earlier applications of the DQ method, Bellman's first method was usually used because it allows the use of arbitrary grid points distribution. However, since the algebraic equation system of this method is ill-conditioned, the number of the grid points usually used is less than 13 [15]. After that, [5] obtain explicit formulations to compute the weighting coefficient for the first and second order derivative. [16] presented a simple algebraic formulation to compute the weighting coefficient of the first order derivative without any restriction on the choice of the grid points and a recurrence relationship to compute the weighting coefficient of the DQ method and [3] and [17] have further 
developed some simple algebraic formulation to compute the weighting coefficient. Recently, the most frequently used DQ procedures to solve one and two dimensional differential equations are Lagrange interpolation polynomials based differential quadrature method (PDQM). The DQ method approximates the derivative of a smooth function at a grid point by a linear weighted summation of all the functional value in the whole computational domain.

The coupled viscous Burgers' equation is a nonlinear partial differential equation of the form

$$
\begin{aligned}
& u_{t}-u_{x x}+\eta u u_{x}+\alpha(u v)_{x}=0, \quad x \in[a, b], t \in[0, T] \\
& v_{t}-v_{x x}+\varepsilon v v_{x}+\beta(u v)_{x}=0, \quad x \in[a, b], t \in[0, T]
\end{aligned}
$$

With initial condition

$$
u(x, 0)=\phi_{1}(x), v(x, 0)=\phi_{2}(x), 0 \leq x \leq 1
$$

The boundary condition

$$
\begin{gathered}
u(a, t)=f_{1}(a, t), \quad v(a, t)=g_{1}(a, t) \\
u(b, t)=f_{2}(b, t), \quad v(b, t)=g(b, t), 0 \leq t \leq T
\end{gathered}
$$

where $\eta, \alpha, \varepsilon$ and $\beta$ are arbitrary constants depending on the system parameters.

\subsubsection{Differential Quadrature Method Based on the Fourier Expansion basis}

Let the first and second order derivative of $u(x)$ at a point $x_{i}$ be approximated by the following equations

$$
\begin{aligned}
& u_{x}\left(x_{i}, t\right)=\sum_{j=1}^{N} a_{i j} u\left(x_{j}, t\right) \text { for } i=1,2, \cdots, N \\
& u_{x x}\left(x_{i}, t\right)=\sum_{j=1}^{N} b_{i j} u\left(x_{j}, t\right) \text { for } i=1,2, \cdots, N
\end{aligned}
$$

where $a_{i j}$ and $b_{i j}$ represents the weighting coefficients of first and second derivative respectively, $N$ is the number of grid points. And the key procedure in $\mathrm{DQ}$ is to determine the weighting coefficient.

For, the solution of any partial differential equation has to be convergent and bounded, so the solution $u(x)$ of the viscous Burger equation be approximated by a Fourier expansion of the form

$$
u(x)=a_{0}+\sum_{k=1}^{N}\left(c_{k} \cos k \pi x+d_{k} \sin k \pi x\right)
$$

$u(x)$ in this equation constitutes an $(N+1)$ dimensional linear vector space since

$$
1, \sin \pi x, \cos \pi x, \sin 2 \pi x, \cos 2 \pi x, \cdots, \sin N \pi x \text { and } \cos N \pi x
$$

are linearly independent vectors.

Thus, we can consider (4.4a) as a set of base vectors.

Actually FDQ uses two sets of base functions, one is the base functions (4.4a) and the other is the terms of the Lagrange interpolating trigonometric poly- 
nomial given by

$r_{k}(x)$

$$
\begin{aligned}
& =\frac{\sin \pi\left(x-x_{0}\right) \sin \pi\left(x-x_{1}\right) \cdots \sin \pi\left(x-x_{k-1}\right) \sin \pi\left(x-x_{k+1}\right) \cdots \sin \pi\left(x-x_{N}\right)}{\sin \pi\left(x_{k}-x_{0}\right) \sin \pi\left(x_{k}-x_{1}\right) \cdots \sin \pi\left(x_{k}-x_{k-1}\right) \sin \pi\left(x_{k}-x_{k+1}\right) \cdots \sin \pi\left(x_{k}-x_{N}\right)} \\
& \quad \text { for } k=1,2,3, \cdots, N . \\
& \quad \text { Let }
\end{aligned}
$$

$$
p(x)=\sum_{k=0}^{N} r_{k}(x)
$$

where

$$
\begin{gathered}
M(x)=\prod_{k=0}^{N} \sin \pi\left(x-x_{k}\right) \\
p\left(x_{i}\right)=\prod_{k=0, k \neq i}^{N} \sin \pi\left(x_{i}-x_{k}\right)
\end{gathered}
$$

By using the above two sets of base vectors to drive explicit formulations to compute the weighting coefficients of the first and second order derivatives, for the non-diagonal weighting coefficient we need to use the second set of base vectors. For simplicity, we set

$$
M(x)=\prod_{k=0}^{N} \sin \pi\left(x-x_{k}\right)=N\left(x, x_{k}\right) \sin \pi\left(x-x_{k}\right)
$$

where

$$
\begin{gathered}
N\left(x_{i}, x_{k}\right)=\prod_{k=0, k \neq i}^{N} \sin \frac{\pi}{2}\left(x_{i}-x_{k}\right)=p\left(x_{i}\right) \\
N\left(x_{i}, x_{j}\right)=N\left(x_{i}, x_{j}\right) \delta_{i j} \text { where } \delta_{i j}=\left\{\begin{array}{ll}
1 & \text { if } i=j \\
0 & \text { if } i \neq j
\end{array}\right. \text { is the Kronecker delta op- }
\end{gathered}
$$
erator.

Then Equation (4.4b) can be reduced to

$$
r_{k}(x)=\frac{N\left(x, x_{k}\right)}{p\left(x_{k}\right)}
$$

Using the same approach as in a polynomial differential quadrature method [18] we let all the base vectors given by Equation (4.10) satisfy Equations ((4.1) and (4.2)) and obtain

$$
\begin{aligned}
& a_{i j}=\frac{N^{\prime}\left(x_{i}, x_{j}\right)}{p\left(x_{j}\right)} \\
& b_{i j}=\frac{N^{\prime \prime}\left(x_{i}, x_{j}\right)}{p\left(x_{j}\right)}
\end{aligned}
$$

It is observed from Equations ((4.11) and (4.12)), the computation of $a_{i j}$ and $b_{i j}$ is equivalent to the evaluations of $N^{\prime}\left(x_{i}, x_{j}\right)$ and $N^{\prime \prime}\left(x_{i}, x_{j}\right)$ since $p\left(x_{j}\right)$ can be calculated by Equation (4.9). We successively differentiate Equation (4.8) to get 


$$
\begin{aligned}
M^{\prime}(x)= & N^{\prime}\left(x, x_{k}\right) \sin \pi\left(x-x_{k}\right)+\pi N\left(x, x_{k}\right) \cos \pi\left(x-x_{k}\right) \\
M^{\prime \prime}(x)= & N^{\prime \prime}\left(x, x_{k}\right) \sin \pi\left(x-x_{k}\right)+2 \pi N^{\prime}\left(x, x_{k}\right) \cos \pi\left(x-x_{k}\right) \\
& -\pi^{2} N\left(x, x_{k}\right) \sin \pi\left(x-x_{k}\right) \\
M^{\prime \prime \prime}(x)= & N^{\prime \prime \prime}\left(x, x_{k}\right) \sin \pi\left(x-x_{k}\right)+3 \pi N^{\prime \prime}\left(x, x_{k}\right) \cos \pi\left(x-x_{k}\right) \\
& -3 \pi^{2} N^{\prime}\left(x, x_{k}\right) \sin \pi\left(x-x_{k}\right)-\pi^{3} N\left(x, x_{k}\right) \cos \pi\left(x-x_{k}\right)
\end{aligned}
$$

From the above equation, we can obtain the following result

$$
\begin{gathered}
M^{\prime}\left(x_{i}\right)=\pi N\left(x_{i}, x_{i}\right)=\pi p\left(x_{i}\right) \\
N^{\prime}\left(x_{i}, x_{j}\right)=\frac{\pi \cdot p\left(x_{i}\right)}{\sin \pi\left(x_{i}-x_{j}\right)} \text { where } i \neq j \\
N^{\prime}\left(x_{i}, x_{j}\right)=\frac{M^{\prime \prime}\left(x_{i}\right)}{\pi} \\
N^{\prime \prime}\left(x_{i}, x_{j}\right)=\frac{M^{\prime \prime}\left(x_{i}\right)-\pi N^{\prime}\left(x_{i}, x_{j}\right) \cos \pi\left(x_{i}-x_{j}\right)}{\sin \pi\left(x_{i}-x_{j}\right)} \text { where } i \neq j \\
N^{\prime \prime}\left(x_{i}, x_{j}\right)=\frac{2}{3 \pi}\left[M^{\prime \prime \prime}\left(x_{i}\right)+\frac{\pi^{3}}{8} N\left(x_{i}, x_{i}\right)\right]
\end{gathered}
$$

Substituting Equations ((4.16) and (4.17)) in to Equation (4.11) we obtain

$$
\begin{gathered}
a_{i j}=\frac{\pi \cdot p\left(x_{i}\right)}{\sin \pi\left(x_{i}-x_{j}\right) \cdot p\left(x_{j}\right)} \text { where } i \neq j \\
a_{i i}=\frac{M^{\prime \prime}\left(x_{i}\right)}{\pi \cdot p\left(x_{j}\right)}
\end{gathered}
$$

Similarly substituting Equations ((4.19) and (4.20)) in to (4.12) and using Equations ((4.17), (4.18)), we obtain

$$
\begin{gathered}
b_{i j}=a_{i j}\left[2 a_{i i}-\pi \cot \pi\left(x_{i}-x_{j}\right)\right] \text { where } j \neq i \\
b_{i i}=\frac{2}{3 \pi}\left[\frac{M^{\prime \prime}\left(x_{i}\right)}{p\left(x_{i}\right)}+\frac{\pi^{3}}{8}\right]
\end{gathered}
$$

From Equations ((4.21) and (4.23)), $a_{i j}$ and $b_{i j}(i \neq j)$ can be obtained.

However, the calculation of $a_{i i}$ from Equation (4.22) and $b_{i i}$ from Equation (4.24) involve the computation of $M^{\prime \prime}\left(x_{i}\right)$ and $M^{\prime \prime \prime}\left(x_{i}\right)$ which are not easy to compute by applying the second set of base vectors $1, \sin (\pi x), \cos (\pi x), \sin (2 \pi x), \cos (2 \pi x), \cdots, \sin (N \pi x), \cos (N \pi x)$ let the vector 1 satisfy Equation (4.1) and (4.2) and then obtain

$$
\begin{aligned}
& \sum_{j=1}^{N} a_{i j}=0 \\
& \sum_{j=1}^{N} b_{i j}=0
\end{aligned}
$$


Using Equations ((4.21), (4.23), (4.25) and (4.26)), the weighting coefficients of the first and second order derivatives can be calculated with FDQ. It should be indicated that these equations can be applied to the periodic problems and the non-periodic problems. For the non-periodic problems, the range in the computational domain is $0 \leq x \leq \pi$, while for the periodic problems, the range in the computational domain is $0 \leq x \leq 2 \pi$.

\subsection{Main Results and Discussion}

The coupled viscous Burgers' Equations (1.1)-(1.3) can be discretized in the Cartesian coordinate system as

$$
\begin{aligned}
\frac{\mathrm{d} u\left(x_{i}, t\right)}{\mathrm{d} t}= & \sum_{j=1}^{N} b_{i j} u\left(x_{j}, t\right)-\eta u\left(x_{i}, t\right) \sum_{j=1}^{N} a_{i j} u\left(x_{j}, t\right)-\alpha\left(u\left(x_{i}, t\right) \sum_{j=1}^{N} a_{i j} v\left(x_{j}, t\right)\right. \\
& \left.+v\left(x_{i}, t\right) \sum_{j=1}^{N} a_{i j} u\left(x_{j}, t\right)\right), x_{i} \in[a, b] \\
\frac{\mathrm{d} v\left(x_{i}, t\right)}{\mathrm{d} t}= & \sum_{j=1}^{N} b_{i j} v\left(x_{j}, t\right)-\varepsilon v\left(x_{i}, t\right) \sum_{j=1}^{N} a_{i j} v\left(x_{j}, t\right)-\beta\left(u\left(x_{i}, t\right) \sum_{j=1}^{N} a_{i j} v\left(x_{j}, t\right)\right. \\
& \left.+v\left(x_{i}, t\right) \sum_{j=1}^{N} a_{i j} u\left(x_{j}, t\right)\right), x_{i} \in[a, b]
\end{aligned}
$$

With initial condition

$$
u\left(x_{i}, 0\right)=\phi_{1}\left(x_{i}\right), v\left(x_{i}, 0\right)=\phi_{2}\left(x_{i}\right), \quad x_{i} \in D
$$

The boundary condition

$$
\begin{gathered}
u(a, t)=f_{1}(a, t), \quad v(a, t)=g_{1}(a, t) \\
u(b, t)=f_{2}(b, t), \quad v(b, t)=g_{2}(b, t), \quad 0 \leq t \leq T
\end{gathered}
$$

where $\eta, \alpha, \varepsilon$ and $\beta$ are arbitrary constants depending on the system parameters.

Where $N$ is the number of grid points, $a_{i j}$ and $b_{i j}$ are the weighting coefficients in the $x$ direction, and when FDQ method is used $a_{i j}$ and $b_{i j}$ are computed by Equations ((4.21), (4.23)). The resulting system of ODEs (4.27) can now be solved by using the classical fourth order Runge-Kutta method.

\subsubsection{Numerical Experiments}

In this section, we consider two examples to test the efficiency of the proposed method. For describing the error, we consider maximum error norm for $u$ defined as follows

$$
\|E(u)\|_{\infty}=\max _{1 \leq j \leq N}\left|u\left(x_{j}, t\right)-u_{N}\left(x_{j}, t\right)\right|
$$

where $u\left(x_{j}, t\right)$ is exact solution of the problem and $u_{N}\left(x_{j}, t\right)$ is the numerical solution at the same mesh points obtained by DQM based on Fourier Expansion basis.

To illustrate the efficiency of the proposed numerical scheme, we solve two test examples and throughout the numerical experiment, we consider step length in time space $\Delta t$. 


\section{Example 1}

Consider the coupled viscous Burgers' equation with the initial and boundary condition taken as follows. $u(x, 0)=\sin (x)$

$$
\begin{gathered}
v(x, 0)=\sin (x), \quad-\pi \leq x \leq \pi, t>0 \\
u(-\pi, t)=u(\pi, t)=0, v(-\pi, t)=v(\pi, t)=0
\end{gathered}
$$

The exact solution taken from [8]

$$
u(x, t)=\mathrm{e}^{t} \sin (x), v(x, t)=\mathrm{e}^{t} \sin (x),-\pi \leq x \leq \pi, t>0
$$

The numerical results are computed with different time step length $\Delta t$ and various values of parameters, $\alpha, \varepsilon$ and $\beta$. The maximum absolute error obtain with $\Delta t=0.01$ up to $\mathrm{T}=3$ are reported in Table 1. It is also shown in Table 2 that absolute errors become reduced when the time step is reduced.

\section{Example 2}

In this problem, we consider the coupled viscous Burger equation with initial conditions

$$
\begin{gathered}
u(x, 0)=a_{0}-2 A\left(\frac{2 \alpha-1}{4 \alpha \beta-1}\right) \tanh (A x) ;-10 \leq x \leq 10 \\
v(x, 0)=a_{0}\left(\frac{2 \beta-1}{2 \alpha-1}\right)-2 A\left(\frac{2 \alpha-1}{4 \alpha \beta-1}\right) \tanh (A x)
\end{gathered}
$$

Table 1. Maximum errors norm of Example 1 for $\eta=-2, \varepsilon=-2, \alpha=1, \beta=1$ at step length $\Delta t=0.01$.

\begin{tabular}{ccc}
\hline$t$ & $\|E(u)\|_{\infty}$ & $\|E(v)\|_{\infty}$ \\
\hline 0.5 & $6.724 \mathrm{E}-4$ & $3.012 \mathrm{E}-3$ \\
1.0 & $7 . .468 \mathrm{E}-3$ & $4.812 \mathrm{E}-4$ \\
2.0 & $5.621 \mathrm{E}-4$ & $5.792 \mathrm{E}-5$ \\
3.0 & $3.460 \mathrm{E}-4$ & $6.182 \mathrm{E}-6$ \\
\hline
\end{tabular}

Table 2. Maximum absolute norm of $u$ and $v$ of Example 1 for different values of $\Delta t$ the proposed method, at $t=1$.

\begin{tabular}{ccc}
\hline$\Delta t$ & $\|E(u)\|_{\infty}$ & $\|E(v)\|_{\infty}$ \\
\hline 0.020 & $1.6121 \times 10^{-5}$ & $2.2234 \times 10^{-5}$ \\
0.0100 & $2.8160 \times 10^{-6}$ & $6.1446 \times 10^{-6}$ \\
0.0050 & $6.9332 \times 10^{-6}$ & $2.8290 \times 10^{-6}$ \\
0.0020 & $7.7126 \times 10^{-7}$ & $3.3328 \times 10^{-7}$ \\
0.0010 & $9.9268 \times 10^{-7}$ & $4.8157 \times 10^{-7}$ \\
0.0005 & $3.1992 \times 10^{-7}$ & $8.8126 \times 10^{-7}$ \\
\hline
\end{tabular}


The exact solution are given by [19]

$$
\begin{aligned}
& u(x, t)=a_{0}-2 A\left(\frac{2 \alpha-1}{4 \alpha \beta-1}\right) \tanh (A(x-2 A t)),-10 \leq x \leq 10, t \geq 0 \\
& v(x, t)=a_{0}\left(\frac{2 \beta-1}{2 \alpha-1}\right)-2 A\left(\frac{2 \alpha-1}{4 \alpha \beta-1}\right) \tanh (A(x-2 A t)),-10 \leq x \leq 10, t>0
\end{aligned}
$$

where $A=a_{0} \frac{4 \alpha \beta-1}{4 \alpha-2}$ and $a_{0}, \alpha, \beta$ are arbitrary constants. In Table 3 and Table 4, we present comparison between the numerical solutions obtained by the present method and the method proposed in literature. Table 3 and Table 4 shows the maximum absolute errors for $\eta=1, \varepsilon=2$ and various values of $\alpha$ and $\beta$.

From Table 1 and Table 2 it can be seen that the solution of Maximum absolute norm $u$ and $v$ decrease to zero at a time $t$ as the value of $\eta, \varepsilon$ increase it can also observed that the Differential quadrature method based on Fourier Expansion basis method is capable of finding numerical solution for larger value of $\eta, \varepsilon$.

Here the parameters $\alpha$ and $\beta$ determine the size of the boundary layer near the edges of the domain. We use the value of $\eta=1, \varepsilon=2$ in our numerical experiment.

Table 3. Comparison of numerical results of Example 2 with the results obtained by [9] and [11] for the variable $u$ with $a_{0}=0.05, N=21$ with $\Delta t=0.01$.

\begin{tabular}{cccccc}
\hline$t$ & $\alpha$ & $\beta$ & $\begin{array}{c}\text { Rashid } \\
\|E(u)\|_{\infty}\end{array}$ & $\begin{array}{c}\text { Jiwari } \\
\|E(u)\|_{\infty}\end{array}$ & $\begin{array}{c}\text { Present Method } \\
\|E(u)\|_{\infty}\end{array}$ \\
\hline 0.5 & 0.1 & 0.3 & $9.619 \mathrm{E}-4$ & $4.173 \mathrm{E}-5$ & $6.812 \mathrm{E}-4$ \\
& 0.3 & 0.03 & $4.310 \mathrm{E}-4$ & $4.585 \mathrm{E}-5$ & $3.312 \mathrm{E}-5$ \\
1.0 & 0.1 & 0.3 & $1.153 \mathrm{E}-3$ & $8.275 \mathrm{E}-5$ & $6.222 \mathrm{E}-4$ \\
& 0.3 & 0.03 & $1.268 \mathrm{E}-3$ & $9.167 \mathrm{E}-5$ & $2.490 \mathrm{E}-4$ \\
& 0.1 & 0.3 & $\ldots$ & $2.408 \mathrm{E}-4$ & $8.612 \mathrm{E}-4$ \\
\hline
\end{tabular}

Table 4. Comparison of numerical results of Example 2 with the results obtained by [9] and [11] for the variable $v$ with $a_{0}=0.05, N=21$ with $\Delta t=0.01$

\begin{tabular}{cccccc}
\hline$t$ & $\alpha$ & $\beta$ & $\begin{array}{c}\text { Rashid } \\
\|E(v)\|_{\infty}\end{array}$ & $\begin{array}{c}\text { Ram Jiwari (2012) } \\
\|E(v)\|_{\infty}\end{array}$ & $\begin{array}{c}\text { Present Method } \\
\|E(v)\|_{\infty}\end{array}$ \\
\hline \multirow{2}{*}{0.5} & 0.1 & 0.3 & $3.332 \mathrm{E}-4$ & $5.418 \mathrm{E}-5$ & $5.428 \mathrm{E}-4$ \\
& 0.3 & 0.03 & $1.148 \mathrm{E}-3$ & $2.826 \mathrm{E}-5$ & $6.867 \mathrm{E}-3$ \\
& 0.1 & 0.3 & $1.162 \mathrm{E}-3$ & $1.074 \mathrm{E}-4$ & $7.890 \mathrm{E}-4$ \\
1.0 & 0.3 & 0.03 & $1.638 \mathrm{E}-3$ & $5.673 \mathrm{E}-5$ & $8.954 \mathrm{E}-4$ \\
& 0.1 & 0.3 & $\ldots$ & $3.119 \mathrm{E}-4$ & $1.126 \mathrm{E}-4$ \\
& 0.3 & 0.03 & $\ldots$ & $1.663 \mathrm{E}-4$ & $7.763 \mathrm{E}-5$ \\
\hline
\end{tabular}


In this case we solve the PDE on the random point distribution. The optimal shape parameter $\alpha$ and $\beta$ is also taken the same as we achieved in the linear PDE case. The error norm in Table 2 shows as differential quadrature method based on Fourier expansion basis. Also works well for this nonlinear problem. However, it is shown that the method can be fluctuated as applied to a wide class of higher-dimension, nonlinear partial differential equations with a little modification depending on the system parameters. In Table 3 and Table 4 we compare the differential quadrature method results with those available ones in the literature. As seen from Table 3 and Table 4 the differential quadrature method based on Fourier expansion is quite comparable with those methods in the literature.

\section{Conclusions and Future Scope}

\subsection{Conclusions}

This study demonstrates the application of FDQ method to solve one-dimensional coupled viscous Burgers' equation. This method produces a system of first order ordinary differential equations, which can be solved numerically by the classical fourth order Runge-Kutta method. To verify the method two test problems have been considered and the results obtained are compared with some other methods which are available in the literature. The numerical solutions obtained show that the present method produces reasonably accurate numerical solution of coupled viscous Burgers' equation.

Therefore differential quadrature method based on Fourier expansion basis is a reliable method to obtain the numerical solution of some physically important nonlinear problems governed by partial differential equation; this is because it brings the problem into a smooth and periodic one.

If the viscous term is dropped from the Burgers' equation the nonlinearity allows discontinuous solutions to develop. A wave is convicting from left to right and solutions for successive times are indicated. Points on the wave with larger values of $u$ convict faster and consequently overtake parts of the wave convicting with smaller values of $u$. Coupled viscous Burgers' equation is the nonlinear partial differential equation, that encounters in the theory of shock waves, mathematical modeling of turbulent fluid and in continuous stochastic processes. It is necessary to postulate a shock across which $u$ changes discontinuously to have a unique solution and so a physically result.

It is shown that the present numerical scheme gives better solutions on most of the grid points. However, it is shown that the method can be fluctuated as applied to a wide class of higher-dimension, nonlinear partial differential equations with a little modification depending on the system parameters.

\subsection{Future Scope}

In the future by applying different differential quadrature method, I will try to solve different partial differential equations numerically. 


\section{Acknowledgements}

We all authors need to pass our heartfelt thanks to Jimma University for funding and supporting this important applicable paper.

\section{Conflicts of Interest}

The authors declare no conflicts of interest regarding the publication of this paper.

\section{References}

[1] Bellman, R. and Casti, J. (1971) Differential Quadrature and Long-Term Integration. Journal of Mathematical Analysis and Applications, 34, 235-238. https://doi.org/10.1016/0022-247X(71)90110-7

[2] Stroud, A.H. and Secrest, D. (1992) Gaussian Quadrature Formulas. Prentice-Hall, Englewood Cliffs, New Jersey.

[3] Shu, C. and Chew, Y.T. (1999) Application of Multi-Domain GDQ Method to Analysis of Waveguides with Rectangular Boundaries. In: Kong, J.A., Ed., Progress in Electromagnetics Research, PIER 21, Electromagnetic Waves, Series 21, EMW, Cambridge, Massachusetts, Chapter 1, 1-18. https://doi.org/10.2528/PIER98052601

[4] Esipov, S.E. (1995) Coupled Burgers' Equations: A Model of Polydispersive Sedimentation. Physical Review E, 52, 3711-3718.

https://doi.org/10.1103/PhysRevE.52.3711

[5] Quan, J.R. and Chang, C.T. (1989) New Insights in Solving Distributed System Equations by the Quadsrature Methods-II. Computers \& Chemical Engineering, 13, 1017-1024. https://doi.org/10.1016/0098-1354(89)87043-7

[6] Quan, J.R. and Chang, C.T. (1989) New Insights in Solving Distributed System Equations by the Quadrature Methods-I. Computers \& Chemical Engineering, 13, 779-788. https://doi.org/10.1016/0098-1354(89)85051-3

[7] Lejeune-Dirichlet, P. (1829) Sur la convergence des séries trigonométriques qui servent à représenter une fonction arbitraire entre des limites données. [On the Convergence of Trigonometric Series Which Serve to Represent an Arbitrary Function between Two Given Limits.] Journal für die reine und angewandte Mathematik, 4, 157-169. (In French) https://doi.org/10.1515/crll.1829.4.157

[8] Kaya, D. (2001) An Explicit Solution of Coupled Burgers' Equations by Decomposition Method. IJMMS, 27, 3711-3718.

[9] Rashid, A. and Ismail, A.I.B.M. (2009) A Fourier Pseudospectral Method for Solving Coupled Viscous Burgers' Equations. Computational Methods in Applied Mathematics, 9, 412-420. https://doi.org/10.2478/cmam-2009-0026

[10] Mittal, R.C. and Arora, G. (2011) Numerical Solution of the Coupled Viscous Burgers' Equation. Communications in Nonlinear Science and Numerical Simulation, 16, 1304-1313. https://doi.org/10.1016/j.cnsns.2010.06.028

[11] Jiwari, R., Pandit, S. and Mittal, R.C. (2012) Numerical Simulation of Two-Dimensional Sine-Gordon Solitons by Differential Quadrature Method. Computer Physics Communications, 183, 600-616. https://doi.org/10.1016/j.cpc.2011.12.004

[12] Séroul, R. (2000) Lagrange Interpolation. In: Programming for Mathematicians, Springer-Verlag, Berlin, 269-273, \$10.9.

[13] Mamo, T., Shiferaw, A. and Jima, M. (2017) Numerical Solution of Burgers' Equation Using Fourier Expansion Based on Differential Quadrature Method. Asian 
Journal of science and Technology, 8, 4760-4768.

[14] Jiwari, R., Mittal, R.C. and Sharma, K.K. (2013) A Numerical Scheme Based on Weighted Average Differential Quadrature Method for the Numerical Solution of Burgers' Equation. Applied Mathematics and Computation, 219, 6680-6691.

https://doi.org/10.1016/j.amc.2012.12.035

[15] Weierstrass, K. (1886) Sur la possibilité d'une représentation analytique des fonctions dites arbitraires d'une variable réelle. Journal de Mathématiques Pures et Appliquées, 2, 105-113, 115-138.

[16] Shu, C. (2000) Differential Quadrature and Its Application in Engineering. Athenaeum Press Ltd., Great Britain. https://doi.org/10.1007/978-1-4471-0407-0

[17] Shu, C. and Xue, H. (1997) Explicit Computation of Weighting Coefficients in the Harmonic Differential Quadrature. Journal of Sound and Vibration, 204, 549-555. https://doi.org/10.1006/jsvi.1996.0894

[18] Pike, J. and Roe, P.L. (1985) Accelerated Convergence of Jameson's Finite Volume Euler Scheme Using Van Der Houwen Integrators. Computers \& Fluids, 13, 223-236. https://doi.org/10.1016/0045-7930(85)90027-1

[19] Soliman, A.A. (2006) The Modified Extended Tanh-Function Method for Solving Burgers-Type Equations. Physica A, 361, 394-404.

https://doi.org/10.1016/j.physa.2005.07.008 